\title{
Duración de la experiencia estética
} Islesia de San Pedro en Klippan, de Sigurd Lewerentz

La relación entre arquitectura y música ha sido temática recurrente en muchos estudios. Silencio y espacio aparecen emparentados, determinados por magnitudes, dilataciones, intervalos: la arquitectura asi entendida sería un problema de vacíos y relaciones, que presta atención a lo que hay entre las cosas antes que las cosas mismas. Y el recorrido seria, tal vez, la manera más adecuada de leer esa arquitectura. Palabras clave: Arquitectura - Suecia, arquitectura - teoría, arquitectura moderna, Lewerentz, arquitectura sacra.

The relationship between architecture and music has been much studied. Silence and space seem related, defined by magnitude, expansion, interval; and architecture in this context is a question of spaces and relationships, of looking at what lies between things not at the things themselves. The promenade is perhaps the best way of reading this architecture. Key words: Architecture - Sweden, architecture - theory modern architecture, Lewerentz, sacred architecture.

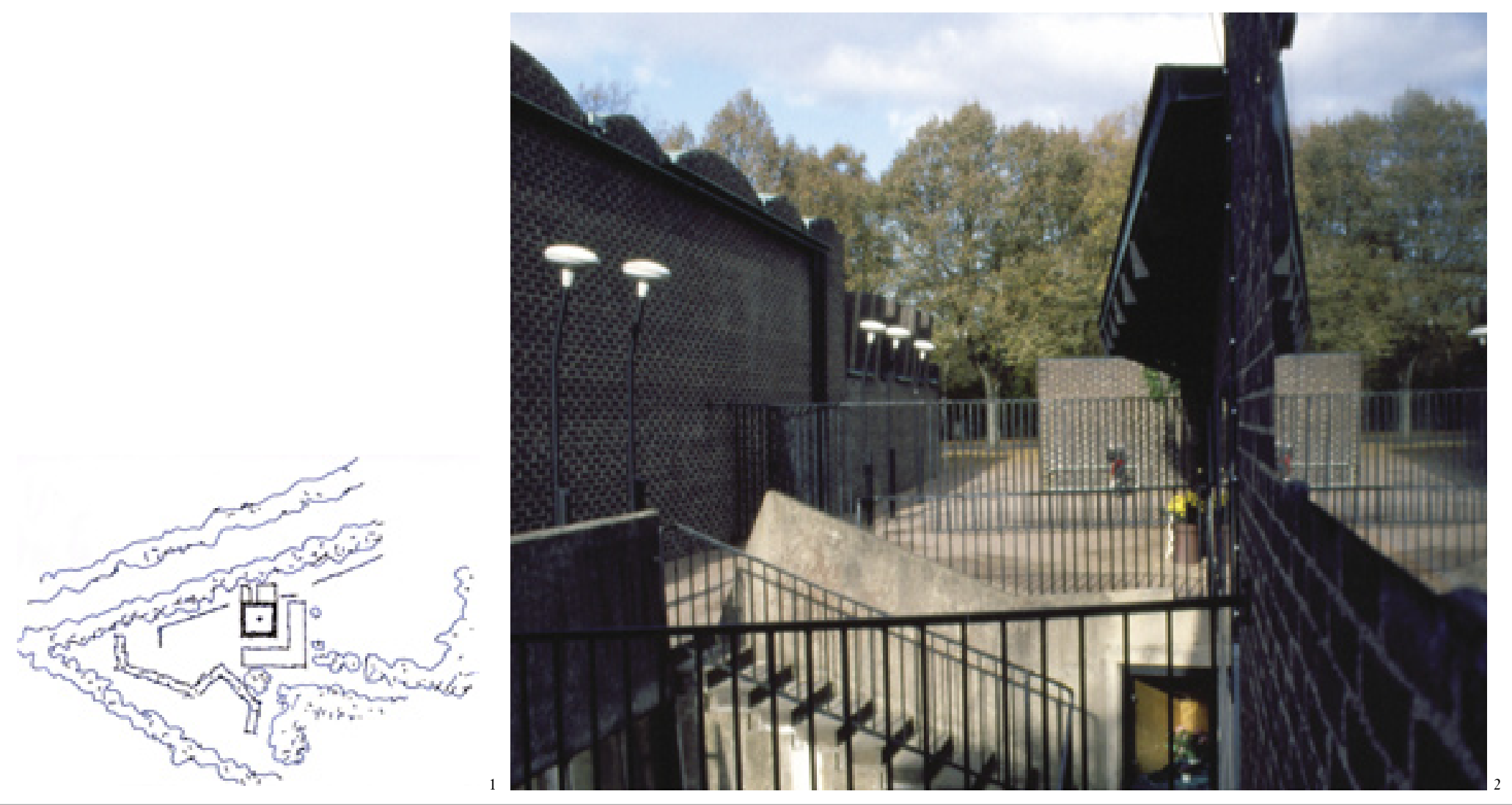

Barcelona, noviembre de 2004

Apreciada Montserrat,

He estado pensando en los múltiples sentidos que la idea de tiempo tuvo y puede tener en el trabajo del arquitecto sueco Sigurd Lewerentz (1885-1975).

Uno es bastante preciso: el que concierne al aparente excesivo tiempo que le tomaba el desarrollo de un proyecto, poniendo a prueba la paciencia y fe de los clientes.

Otro, no menos interesante: las sucesivas intervenciones en una misma obra, como puede ser el caso de los cementerios que llevó a cabo, obras que por su propia naturaleza tienen que ir adaptando su capacidad desde unas posibilidades tanto económicas como físicas.

Desde una vertiente más fenomenológica y general, también interesa el tiempo que tomamos en experimentar la obra. Aqui toma especial énfasis la idea de recorrido como medio de aprehender la obra. En arquitectura, el recorrido no sólo constituye un becho sino que también es conocimiento. Ligado a lo anterior aparece la resistencia o persistencia de la obra a lo largo de un periodo. Este sentido también se refiere a una resistencia atmósférica, la capacidad del edificio de no sólo resistir el paso del tiempo sino también de su capacidad de dar cobijo, protección y asiento a determinadas funciones y actos. En esta oportunidad me pareció interesante presentar una obra de Lewerentz desde una vertiente fenomenológica producto de la visita a la iglesia de Klippan, en el sur de
Suecia. Es prácticamente la última obra de Lewerentz, en la que resuenan, se desarrollan y decantan temas que sostuvo en el tiempo. Uno de estos temas fue la preocupación por el rito que los lugares tenían que permitir, dando importancia a los pasos, al modo en que estos lugares tenían que ser recorridos, experimentados, conocidos. El edificio-objeto se convierte en una partitura a interpretar por el sujeto.

Cuando pienso en la iglesia de Klippan desde la interpretación me vienen a la mente las dos versiones que Glenn Gould bizo de las Variaciones Goldberg de 7. S. Bach: la primera, de 1955, dura 38 minutos y 26 segundos; la segunda, de 1981, 51 minutos y 14 segundos, casi 13 minutos más.

La gran lección que Gould nos enseña es que cuando en música se eliminan los accidentes, "el tiempo se dilata como un océano" (A. Fancelli). El fenómeno no sólo se explica por una cuestión de ralentización, sino que en la segunda versión cada nota está pesando por ella misma: no hay distinción entre las notas de paso y las reales, todas ocupan el mismo plano. De hecho, no bay mucha diferencia en los tempi: es pura apertura de espacio, no problema de la velocidad. De alguna forma se puede decir que en la segunda el protagonista es el vacío, el intervalo, lo que dura el ir de una nota a otra. Así esta arquitectura sacra de Lewerentz.

Gracias por esta oportunidad y espero sirva. Un abrazo,

Fosé Quintanilla Chala. 
"Ser arquitecto significa, simplemente, ser capaz de condensar el tiempo basta convertirlo en un instante, en un lugar concreto". R. Moneo

"La verdad es que el tiempo, de todos los caracteres de la realidad, es el menos real". X. Zubiri

En 1962, se encarga a Lewerentz el diseño de una nueva iglesia para la pequeña comunidad industrial de Klippan en la provincia de Escania, Suecia.

Dos son los temas con que Lewerentz se aproxima a esta obra. Por un lado, la interpretación de la Sagrada Escritura, planteando la forma en función de la liturgia: el altar se sitúa en el corazón de la asamblea, en una configuración de circulo abierto que corresponde al término circumstantes con que Lewerentz explicó el proyecto.

Por otro, el sistema y proceso constructivos, y las interminables revisiones in situ. Lewerentz define un material, el ladrillo, y se da reglas que aplica rigurosamente: lo usará para todos los propósitos (pavimento, muro, bóveda, altar, púlpito, asiento); usará sólo ladrillo standard, no habrá formas especiales y ningún ladrillo será cortado. La única manera de reconciliar estas tres condiciones es mediante una relación muy libre con el mortero de junta. Lewerentz situó el conjunto parroquial en el extremo occidental del principal parque de Klippan, cerca del ángulo donde convergen dos avenidas principales, en el límite entre lo urbano y lo rural. La lectura total del complejo nunca es posible, se va descubriendo en un progresivo acercamiento al edificio. Con la presencia del volumen en el parque volvemos a redescubrir articuladamente el paisaje. Es a través de una multiplicidad de encuadres como conseguimos hacernos una idea del edificio y del lugar. A esto contribuye la autonomía del volumen del santuario que podemos circundar completamente. El principio de circumstantes también actúa a escala del paisaje (fig. 1). La ausencia de todo elemento vertical crea una fuerte impresión de estabilidad. En esta obra Lewerentz ha renunciado a todo elemento vertical pero no a la verticalidad, a todo reconocimiento geométrico, pero no a su rigor. Desde el exterior es imposible reconocer el cuadrado de la planta del santuario. El interior sólo se expresa en los recortes que las bóvedas producen en el muro. Aquí, el perfil del volumen redibuja el follaje de los árboles contiguos (fig. 2).

Una vez traspasado el vestíbulo de acceso, lo primero que reconocemos es la luz deslumbrante de un par de ventanas. Lo cegador de su presencia nos impide cualquier visión hacia el exterior cargando de gravedad la densa penumbra interior. Pronto descubrimos que estas ventanas están iluminando o mejor dicho, barriendo la penumbra en torno a una transfigurada pila bautismal. Simultáneamente percibimos el movimiento del suelo, que se eleva en la zona bautismal mientras el resto desciende hacia la zona del altar (fig. 3).

En esta espesa oscuridad interior sólo cabe la exploración y la proximidad a las cosas.

Como en otras ocasiones, Lewerentz nos sitúa ante lo inesperado. Ningún aspecto de la obra se puede dar por sentado, ya sea en la implantación o en el detalle. Lewerentz traza un simple cuadrado en planta para comenzar a trabajar otro tipo de riquezas, por ejemplo, la pendiente. Lewerentz recrea un nuevo paisaje artificial en el interior de este espacio, cuyo propósito pareciera consistir en hacer sensibles los sentidos.

Esto es llevado incluso al modo en que plantea los elementos que conforman el espacio. El techo, por ejemplo, en su dinámica apariencia nos resulta una alegoría al cambiante cielo nórdico (fig. 4). El suelo define zonas y recorridos, no sólo por la uniformidad del material que lo constituye, sino también por los pautados que definen la posición de los ladrillos y las juntas de mortero (fig. 5). Esto es apoyado por los puntos de luz, mientras la pendiente en el plano se inclina hacia el altar; un efecto que es más

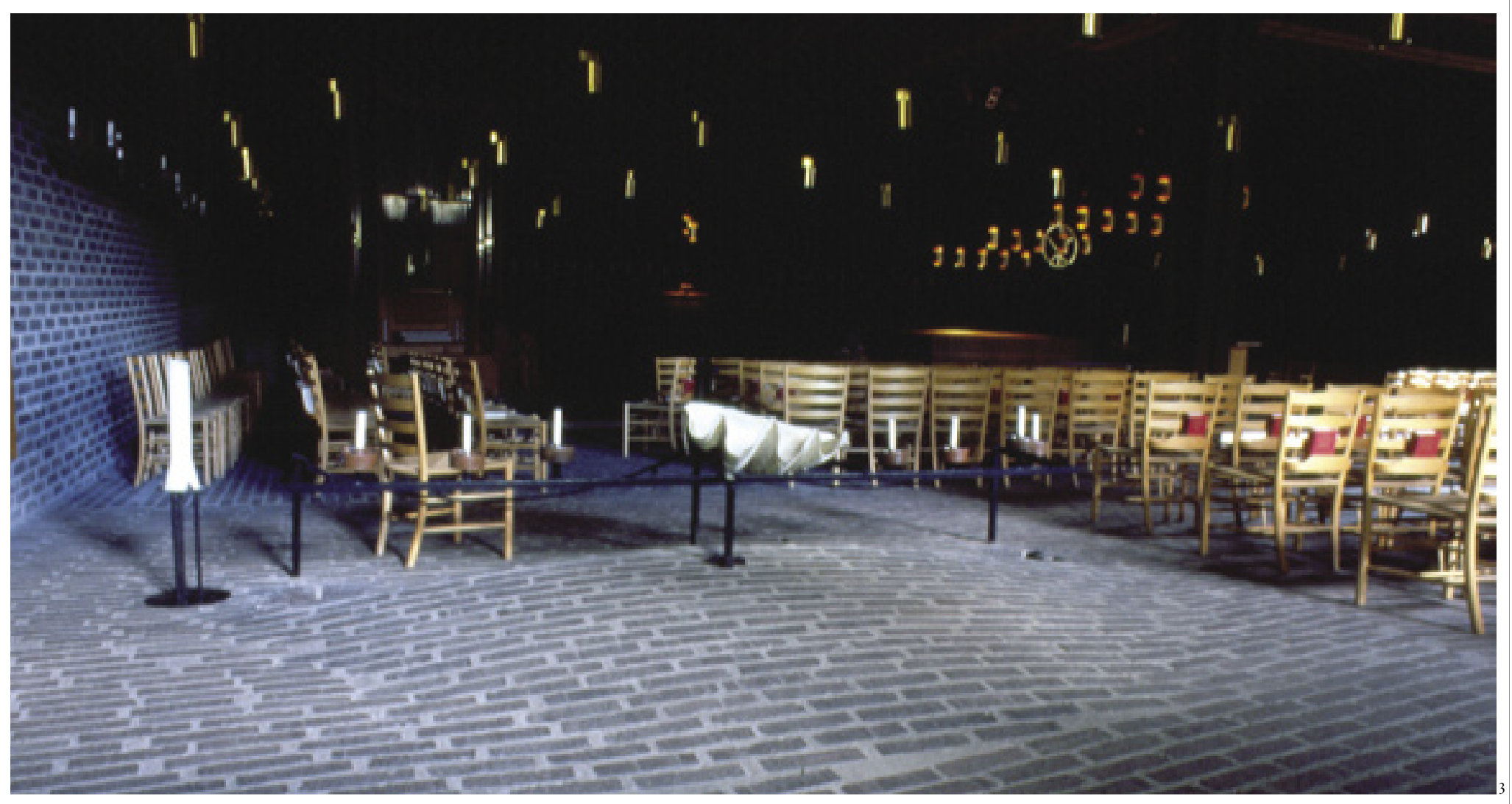


sentido que visto debido a lo escaso de la luz. La equivalencia entre largo y ancho de la sala permite dar un mayor protagonismo a la reunión de la asamblea. Debido a esta configuración estática y centralizada, en el Santuario todos los elementos se muestran al mismo tiempo.

Algunos procedimientos compositivos convierten la pila bautismal, un elemento fundamental de la liturgia cristiana, en un lugar notable: su posición con respecto al total, los elementos que la componen, el trabajo del pavimento en torno a ella, el modo en que está iluminada naturalmente, la acción del goteo incesante hacia una fuente cuyo fondo es imperceptible (fig. 6). El altar, desde su avanzada posición con respecto a la nave y el púlpito desde su posición de retiro, constituyen lugares, focos en el interior del santuario (fig. 7).

De esta manera, el espacio centralizado del santuario es activado por una serie de focos que están en tensión con la posición del altar, a la vez que ellos mismos constituyen un centro.

$\mathrm{El}$ interior es un espacio policéntrico. No encontramos soluciones constructivas convencionales. Cada elemento ha sido pensado de nuevo no sólo técnicamente sino también en su posición con respecto a los demás. Entre las soluciones constructivas no convencionales destacan las ventanas fijas a la cara exterior de los muros (fig. 8), o la síntesis estructural del único pilar con que resuelve el soporte de la cubierta que trasciende su función primera para convertirse en una figura central de la liturgia: la cruz (fig. 9).

Libertad y condensación definen esta arquitectura en que, al igual que sucede con la palabra en el verso, cada elemento es un compromiso. Dicho de otro modo, Lewerentz ha neutralizado la distinción entre figura y fondo, soltando los elementos para volver a ensamblarlos. Incluso el simple mortero de junta adquiere un papel sustancial: ajustar el material ladrillo con el espacio (fig. 10). De este modo en Klippan una profunda calma invade el interior del santuario. Una soledad conmovedora habita su interior alterada por el sonido del puntual goteo de la pila bautismal sobre la imperceptible fuente al fondo del pavimento. Cada luminosa gota va a perderse en la oscuridad de un fondo que percibimos sin medida.

Una soledad sonora, ritmada, expectante, como si una presencia desconocida estuviera contando cada uno de los ladrillos y fuera a detenerse al llegar al último. Pero a fuerza de unidad, aquí no hay primeros ni últimos, sólo simple transcurrrir. Todo en este espacio está equilibrado. El interior podrá iluminarse más o menos pero incluso este hecho tiene un límite: no más luz que sombra. Equilibrio no jerárquico.
Lo iluminado viene del paisaje, no del interior de la habitación sino del interior del paisaje que es donde hay que tener puestos los ojos, la voluntad, el corazón. Una cierta sensación de que todo aún está siendo creado lo invade todo. Quizá sea ésta la única y gran lección pastoral que un creyente puede sacar de esta experiencia de espacio desprovisto, austero, en definitiva protestante.

Como si fuera lugar de peregrinación, este objeto está solo en el paisaje, oscuro, repeliendo, rechazando la visión con sus apaisadas ventanas que orientadas al parque actúan de espejo, condensando distancias, insistiendo en la idea que ahí, en el edificio, no encontraremos nada (fig. 11).

Un gran estanque a la salida vuelve a ser un espejo, esta vez horizontal, donde se reflejan el cielo, las nubes y el sol de la tarde. Desde la profundidad por la que hemos transitado por primera vez, en el crepúsculo de la jornada, nos encontramos con la altura. Y sobre la oscura fachada y las oscuras puertas de salida del templo, el sol y su reflejo se encargan de transmutar su sombría apariencia (fig. 12).

Lewerentz en esta obra ha reducido el léxico con que trabajar. Arriesgando una hipótesis podríamos decir que en esta obra Lewerentz está con el mismo grado de entereza, con el mismo dominio del lenguaje, con el mismo conocimiento y conciencia
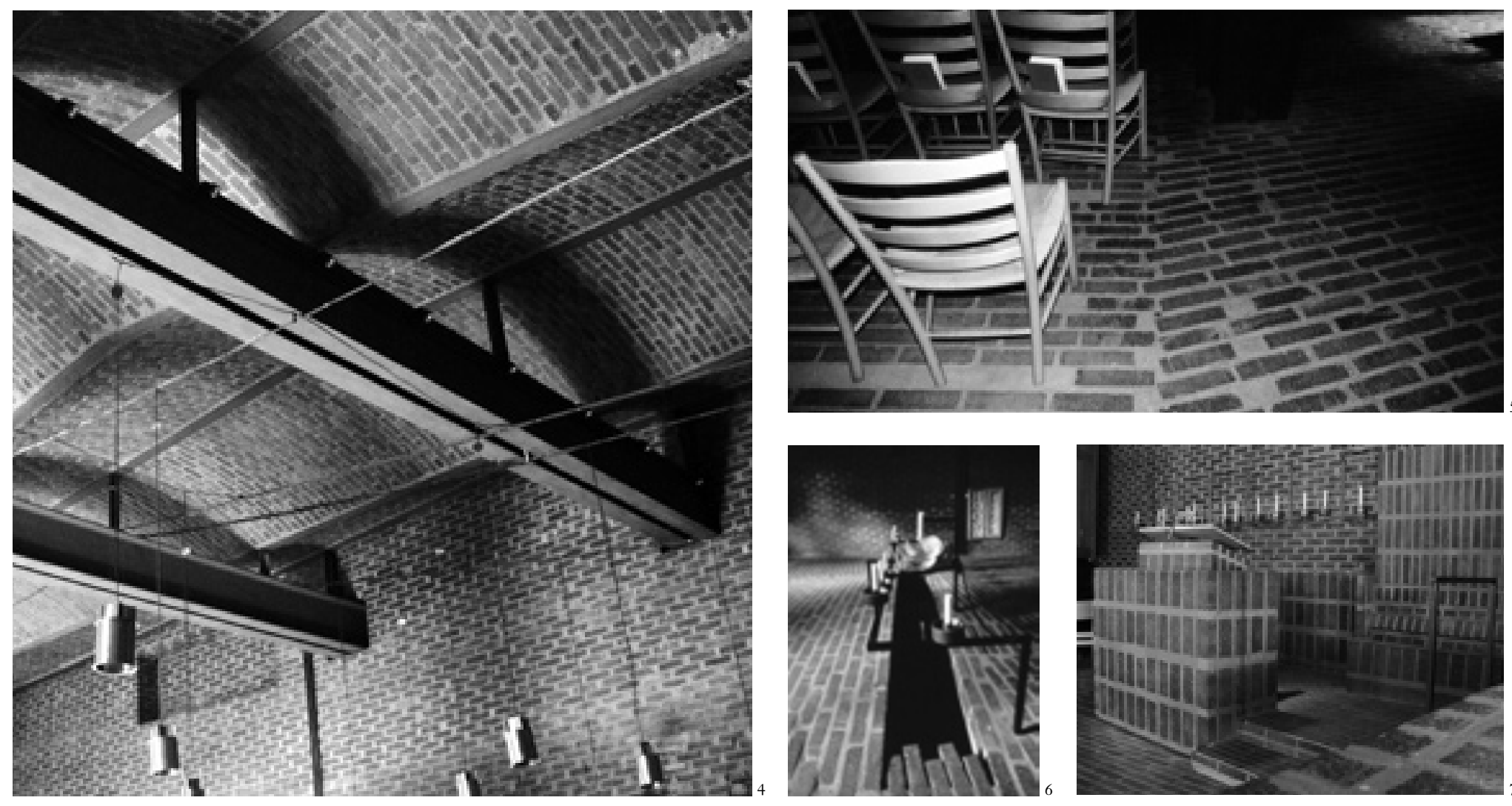

ARQ Ensayos y documentos Essays and documents 
de las leyes que gobiernan la producción de la forma, que cuando abordó la Capilla de la Resurrección en el Cementerio del Bosque (1921-25). Ahí también los elementos eran los suficientes.

Pero esta no es la única referencia que se puede hacer a la Capilla de la Resurrección: una matriz idéntica define el modo en que el espacio es recorrido, aunque las finalidades son diferentes. El rito de paso que ensayara Lewerentz en sus primeros proyectos, una vez conquistado como mecanismo organizativo, es depurado y aplicado en cada obra de arquitectura sacra. Rito y forma.

En la búsqueda de una belleza densa y salvando las marcadas diferencias, la Capilla de la Resurrección es también una fuente para ciertas ideas que Lewerentz explorará en sus últimos edificios religiosos, como puede ser el tratamiento de cada elemento como una pequeña unidad, una pequeña obra en sí misma, no sometida a sistema o canon que la norme.

Sin embargo, el cambio de sensibilidad en esta obra, a través de la entrada de nuevos criterios de proyecto, ha producido que los elementos se hayan constituido en lugares. El espacio está construido con lo indispensable para encontrar lo absoluto.

En el vacío de santuario de Klippan cada elemento está pesando por sí mismo. También la penumbra y el silencio. ARQ

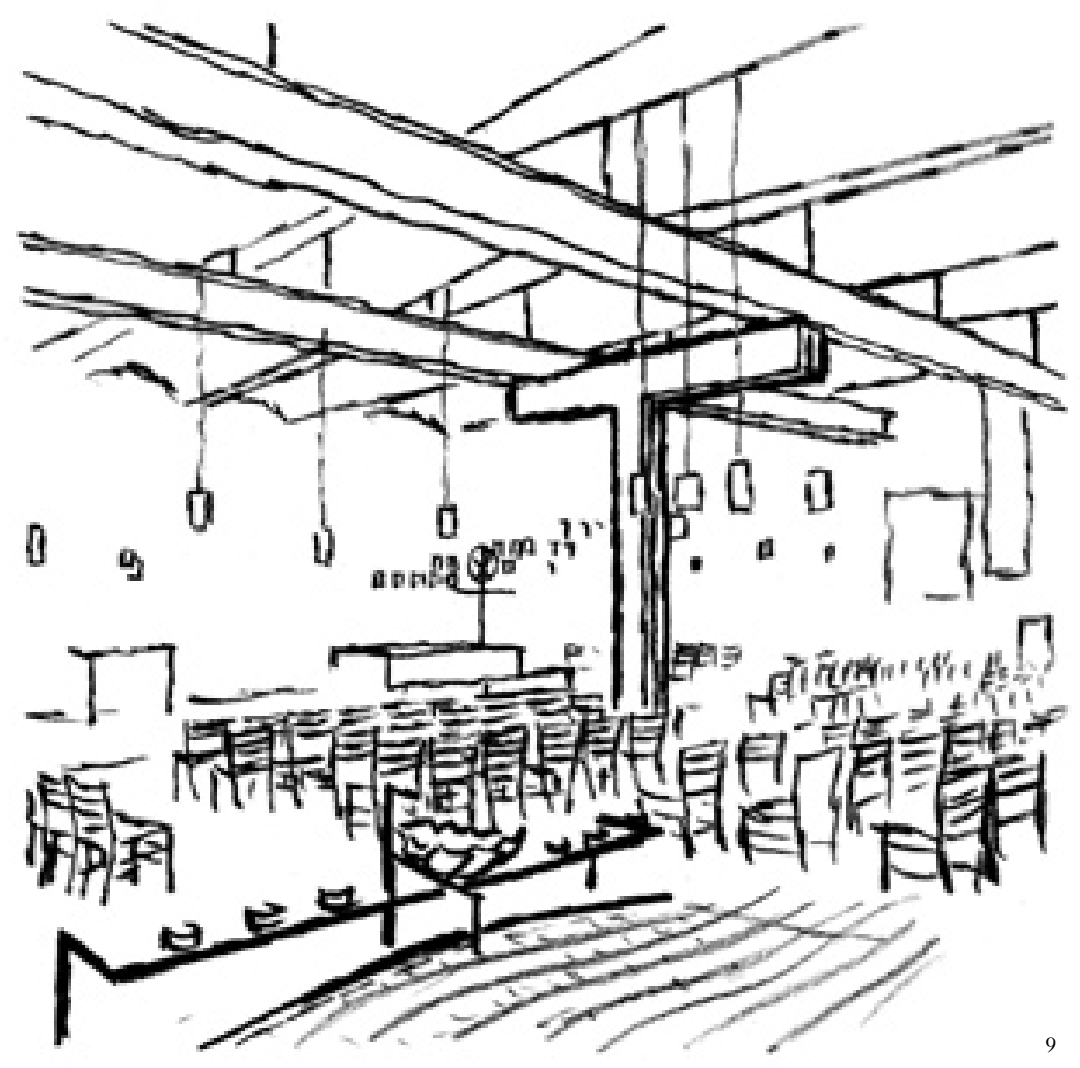

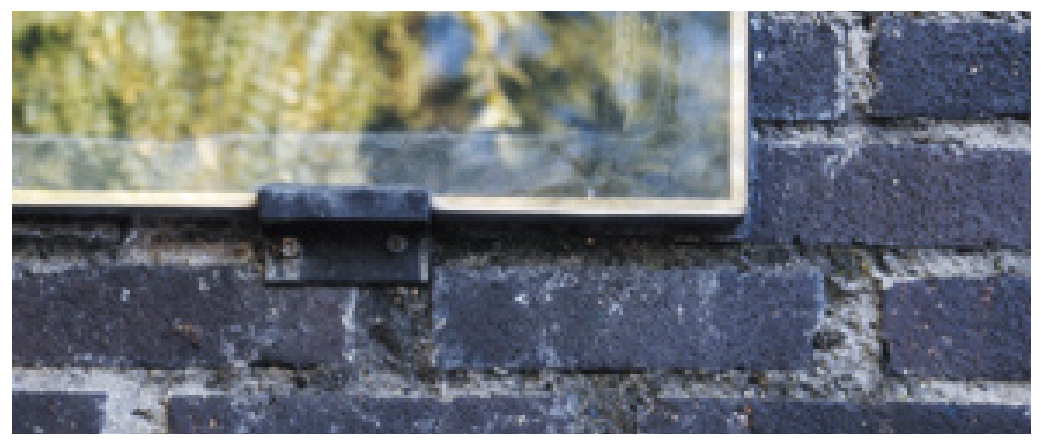
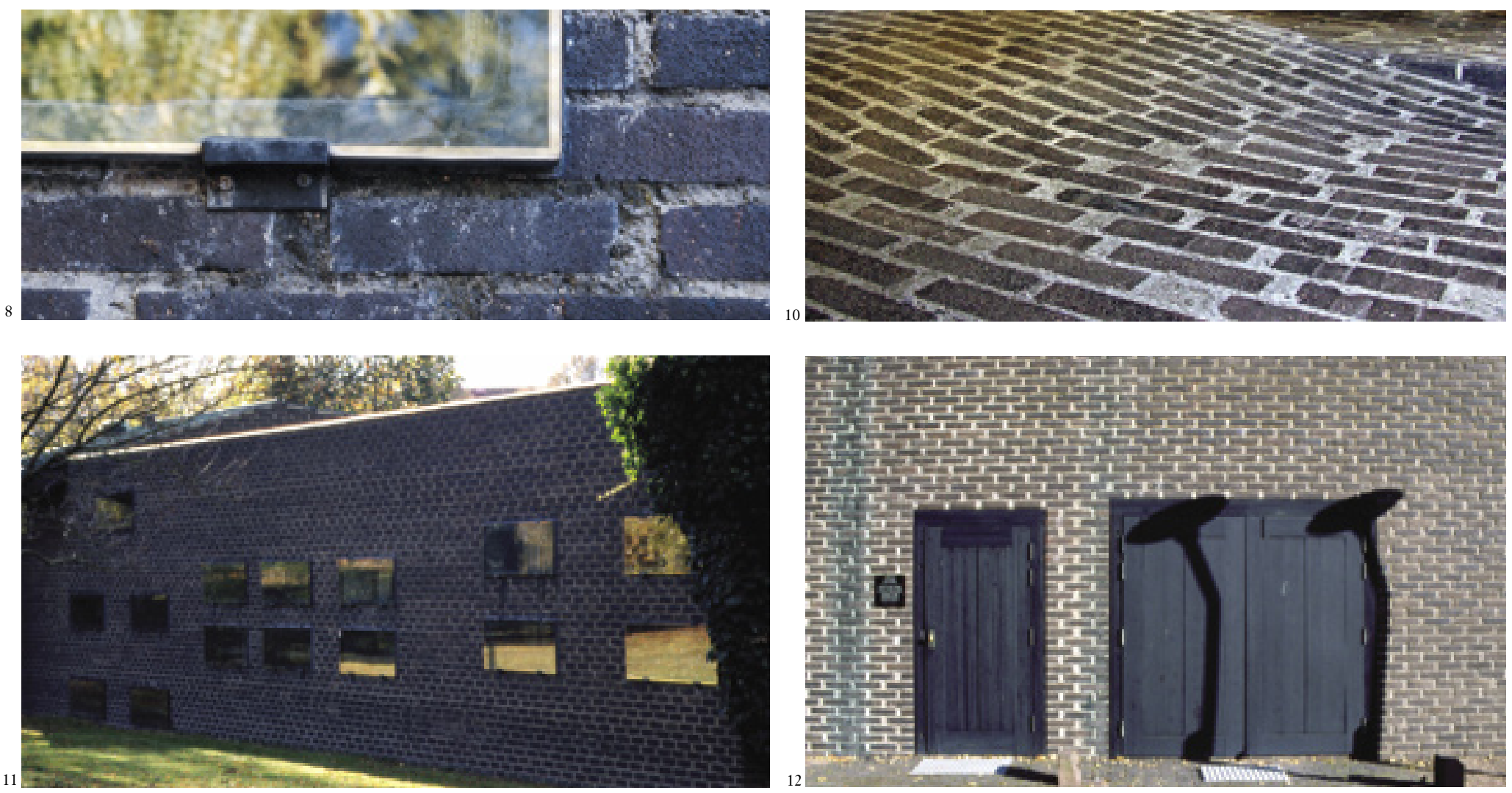